\begin{tabular}{|l|l|c|c|}
\hline Eiszeitalter u. Gegenwart & 36 & $89-109$ & Hannover 1986 \\
& 7 Abb., 2 Tab. & \\
\hline
\end{tabular}

\title{
Geomorphologische Lage, Deckschichten und Molluskenführung letztinterglazialer Hochflutlehme im Neckartal (Baden-Württemberg)
}

\author{
ERHARD Bibus \& WolfGang RÄHLE *)
}

Riß glaciation, terraces, Eemian high-flood loams, Würmian, loess, paleosoils, mollusc faunas, valley formation Southwest German Hills (Rottenburg, Heilbronn-Böckingen), Baden-Wurttemberg TK 25: Nr. 6821, 7519

\begin{abstract}
Kurzfassung: Von zwei Stellen des Neckartales werden rißzeitliche Terrassenreste mit abschließenden eemzeitlichen Hochflutlehmen und würmzeitlichen Deckschichten beschrieben. Aufgrund der Molluskenführung und des Ausbildungsgrades der Paläoböden handelt es sich um Hochflutlehme, die verschiedene Abschnitte des letzten Interglazials repräsentieren. Die durch paläontologische und paläopedologische Methoden erzielten Ergebnisse lassen sich sehr gut korrelieren. Aus der geomorphologischen Lage der Hochflutlehme werden Rückschlüsse über die jungpleistozäne Talentwicklung gezogen, die von früheren Auffassungen z. T. erheblich abweichen.
\end{abstract}

[Geomorphological Site, Superficial Layers and Mollusc Structures of Last Inter-Glacial High-Flood Loams in the Neckar Valley (Baden-Württemberg)]

Abstract: Terrace relicts from the Riss Period with terminal high-flood loams and superficial layers from the Wurm Period are described for two sites in the Neckar Valley. Due to the mollusc layers and the stage of development of the palaeosoils, one can state that the high-flood loams represent various sections of the last inter-glacial period. The results from palaeontological and palaeopedological analyses show a high degree of correlation. Conclusions, which differ greatly from previous results, have been drawn concerning the Late Pleistocene valley formation by means of a comparison of the geomorphological sites of the high-flood loams.

\section{Einleitung}

Die geomorphologische Entwicklung des Neckartales wurde bereits in unterschiedlichen Flußabschnitten

*) Anschrift der Autoren: Prof. Dr. E. BiBuS, Geographisches Institut der Universität Tübingen, Hölderlinstraße 12 , D - 7400 Tübingen 1; Dr. W. RÄHLE, Institut für Biologie III der Universität Tübingen, Morgenstelle 28, D - 7400 Tübingen 1. untersucht, ohne daß bis zum heutigen Zeitpunkt eine zusammenfassende Darstellung der gesamten Talentwicklung erfolgt ist. Häufig standen nur die breit ausgebildeten älteren Terrassen im Blickpunkt, während den tiefer liegenden Terrassenkörpern nur wenig oder gar keine Aufmerksamkeit geschenkt wurde (vgl. z. B. HeLD 1913; MüHLFELD 1953). Als wohl differenziertester Versuch einer Terrassengliederung muß die Untersuchung von ACHILLES (1939) angesehen werden, in der auch ansatzweise die Grobgliederung der Deckschichten zur stratigraphischen Einstufung eine Berücksichtigung fand. Auffallend ist jedoch, daß, abgesehen von Terrassenvorkommen mit hangenden Travertinen im Cannstätter Raum (REIFF 1965, 1973), sichere Terrassendatierungen selbst in jüngeren Arbeiten (HELLER 1961; MADER 1978) nicht vorgelegt wurden. Es überrascht deshalb nicht, wenn bereits die Höhenlage der Rißterrasse über der Talaue sehr unterschiedlich angegeben wird (z. B. BÖHM 1927, $30 \mathrm{~m}$ im obersten Neckartal, $70 \mathrm{~m}$ unterhalb von Rottweil; SCHMIDT 1924, 1932, 20-25 m bei Rottenburg; ACHILLES 1939, 6-19 m im mittleren Neckartal; Heller 1961, 20-25 m zwischen Tübingen und Plochingen; REIFF 1973, 5-6 m bei Bad Cannstatt). Besonders erwähnenswert sind jedoch die von MADER (1978) z. T. in Anlehnung an BÖHM (1927) mitgeteilten Taleintiefungsraten. So soll sich der Neckar in der Umgebung von Rottenburg seit dem Mindel/Riß-Interglazial um $70 \mathrm{~m}$, bei Oberndorf um $240 \mathrm{~m}$ und seit der Würmkaltzeit bei Sulz um $120 \mathrm{~m}$ eingeschnitten haben. Dagegen werden 90-100 m über der Talaue liegende Sedimente von anderen Autoren (EISSELE \& SCHÄDEL 1957; HELLER 1961) in der Umgebung von Tübingen mindestens an die Grenze Pliozän/Pleistozän gestellt.

Bei solchen Diskrepanzen schien es angebracht, zunächst in einem ersten Schritt die geomorphologische 
Position der letztinterglazialen Hochflutlehme und der Rißschotter zu erfassen und durch paläopedologische und malakologische Befunde abzusichern. Die Untersuchung der Schnecken schien auch deswegen interessant zu sein, da molluskenführende letztinterglaziale Sedimente in Baden-Württemberg, wenn man von den Travertinen von Bad Cannstatt und den Kalktuffen von Dießen und Dettingen bei Horb absieht, bisher kaum bearbeitet wurden.

Nachfolgend werden Befunde aus Aufschlüssen bei Rottenburg und Heilbronn-Böckingen mitgeteilt (vgl. Abb. 1), die sich für die angeschnittene Frage als besonders günstig erwiesen haben.

\section{Die Aufschlüsse an der Osttangente von Rottenburg}

Beim Bau der Umgehung von Rottenburg war nordöstlich des Friedhofs „Sülchen” der Talhang über der Neckaraue durch Hanganschnitte, Brückenbauwerke und Sondierungsbohrungen gut aufgeschlossen. Die durch Rinnen gegliederte Taulaue liegt östlich von Rottenburg zwischen 338 und 343 m üb. NN und weist aufgrund unterschiedlich ausgebildeter Böden (Braune kalkhaltige Auenböden, Schwarzerden) eine Zweigliederung auf. Über den randlichen Talauenschottern, auf denen Reste des mittelalterlichen Rottenburg ausgegraben wurden, folgen zwei Neckarterrassen (vgl. Abb. 2). Während die tiefere Terrasse durch mehrere Meter mächtige Deckschichten so stark verkleidet ist, daß sie sich im Relief nicht abzeichnet, tritt die obere Terrasse als schmale Schotterverebnung morphologisch deutlich in Erscheinung. Weit verbreitet sind im Raum Rottenburg außerdem noch ca. $60 \mathrm{~m}$ über der Talaue liegende Kiese, die an der „Zangenhalde” bei 395 m üb. NN dem Gipskeuper auflagern. Die teilweise rötlich verwitterten und lokal durch Kalk verbackenen Kiese waren auch unter Lößdeckschichten in der Trasse der Umgehungsstraße aufgeschlossen. Die engräumige Staffelung der tieferen Terrassen läßt vermuten, daß eine weitgehend lückenlose Abfolge der talauennahen Schotterkörper vorhanden ist. Aufgrund der Deckschichten eignet sich vor allem die untere Terrasse für stratigraphische Untersuchungen.

Die gut geschichteten und vorwiegend aus Muschelkalk bestehenden Neckarschotter waren im Brücken-

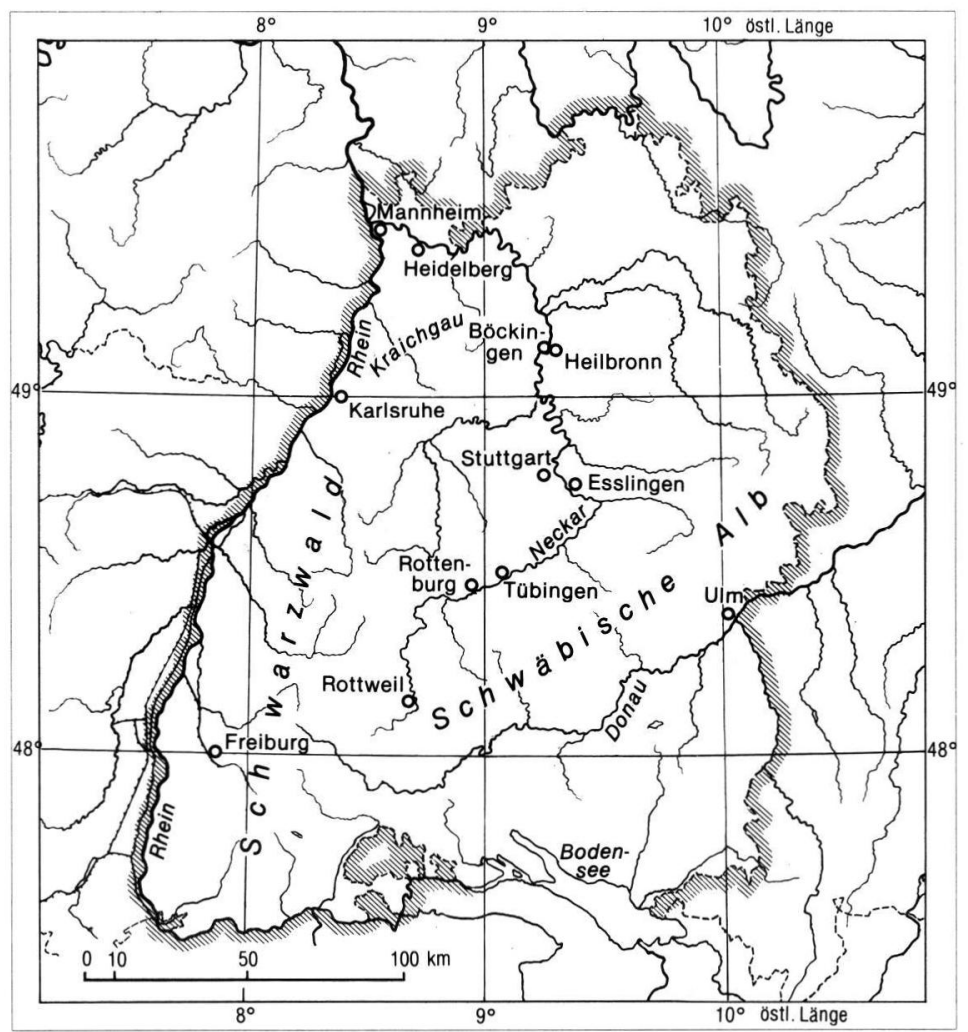

Abb. 1: Übersichtskarte mit Einzugsgebiet des Neckars. 


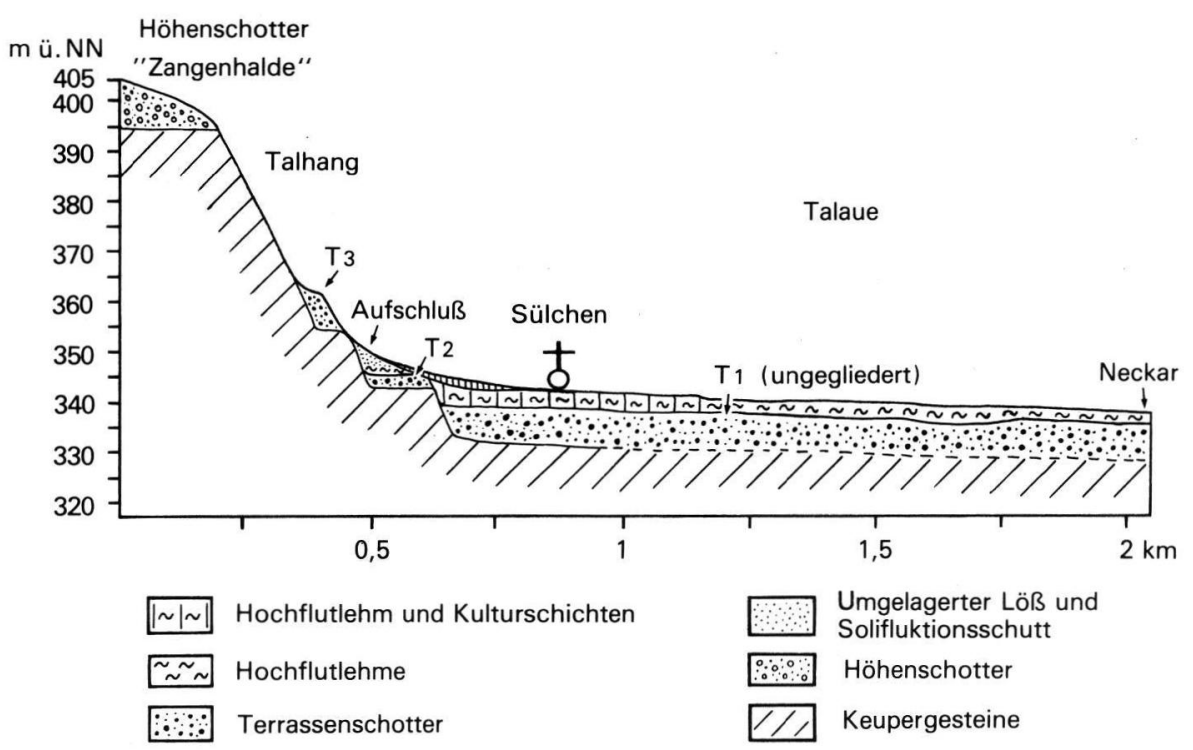

Abb. 2: Terrassenabfolge am nordöstlichen Stadtrand von Rottenburg.

bauwerk an der alten Landstraße nach Wurmlingen mit der Obergrenze bei $345 \mathrm{~m}$ üb. NN aufgeschlossen. Nach den durchgeführten Bohrungen handelt es sich um einen 2,5-3 m mächtigen Schotterkörper nahe dem ehemaligen Talrand, da in einer hangwärts gelegenen Bohrung des Brückenbauwerks der Kies nicht mehr erfaßt werden konnte.

Der Schotterkörper wurde von einem in einer flachen Rinne liegenden Hochflutlehm überlagert, der im Zentrum der Mulde eine Mächtigkeit von 3 m erreichte (vgl. Abb. 3, Schicht 10). Im tiefsten Bereich war der kalkhaltige Hochflutlehm sandig ausgebildet und lokal von grauen Tonschmitzen durchsetzt. Mit zunehmender Profilhöhe fand ein Korngrößenwechsel zu einem feinsandigen bis schluffigen Lehm statt, der intensiv rostbraun-fahlrötlich-grau marmoriert war und bis $10 \mathrm{~cm}$ große Kalkkonkretionen führte. Es handelte sich um einen $\mathrm{G}_{\text {or }} \mathrm{C}_{\mathrm{c}}$-Horizont eines Auenbodens, in dem neben Lößlehm wohl auch Keupermergel aufgearbeitet worden sind. Im oberen Bereich war der Hochflutlehm verbraunt, so daß als Boden ein Braunerde-Gley vorlag. Für eine stärkere pedogene Überprägung des $60 \mathrm{~cm}$ mächtigen $\mathrm{G}_{\mathrm{o}} \mathrm{B}_{\mathrm{v}}$-Horizontes sprach auch eine $30 \mathrm{~cm}$ starke Entkalkung. An der Untergrenze des entkalkten Verbraunungshorizontes setzte der intensiv ausgebildete $\mathrm{C}_{\mathrm{c}}$-Horizont ein. Abgesehen von den entkalkten Partien führte der gesamte Hochflutlehm Schnecken, die vor allem in den basalen Ton- und Sandlagen partienweise sehr angereichert sein konnten. Außerdem wurde $30 \mathrm{~cm}$ über der Rinnenbasis die linke Geweihstange von Cervus elaphus L. 1758 (Rothirsch) $^{1}$ ) gefunden.

Aus den geschilderten Verhältnissen läßt sich folgende Entwicklung rekonstruieren:

Nach Ablagerung des im damaligen Auenrandbereich geringmächtigen Schotterkörpers erfolgte aus Rinnen eine Überschüttung mit Hochflutlehm. Die Aufschüttungstendenz wurde durch eine geringe Eintiefungsphase mit einer schwachen Absenkung des Grundwasserspiegels unterbrochen. Neben den Vergleyungseinflüssen konnte nunmehr zusätzlich eine Entkalkung und deutliche Verbraunung einsetzen. Es entstand ein Boden, der vom Ausbildungsgrad wesentlich kräftiger als alle interstadialen Böden im Würm- und Rißlöß (vgl. z. B. Semmel 1968; BiBuS 1974) entwickelt ist. Andererseits wies der Boden wegen seiner fehlenden Lessivierung nicht die kennzeichnenden Merkmale einer voll ausgereiften interglazialen Bildung auf. Allerdings ist eine nachträgliche Kappung höherer Profilabschnitte nicht auszuschließen. Wenn man von einer solchen Möglichkeit, die zwar sehr wahrscheinlich ist, absieht, dann läßt sich aus den erhaltenen Relikten immer noch eine Entstehung des Hochflutlehms und des Braunerde-

1) Für die Bestimmung des Fundes danke ich Herrn Priv.-Doz. Dr. W. v. Koenigswald, Darmstadt, und für die Arbeiten bei der Bergung und Präparation Herrn LUGINSLAND, Tübingen, recht herzlich. 


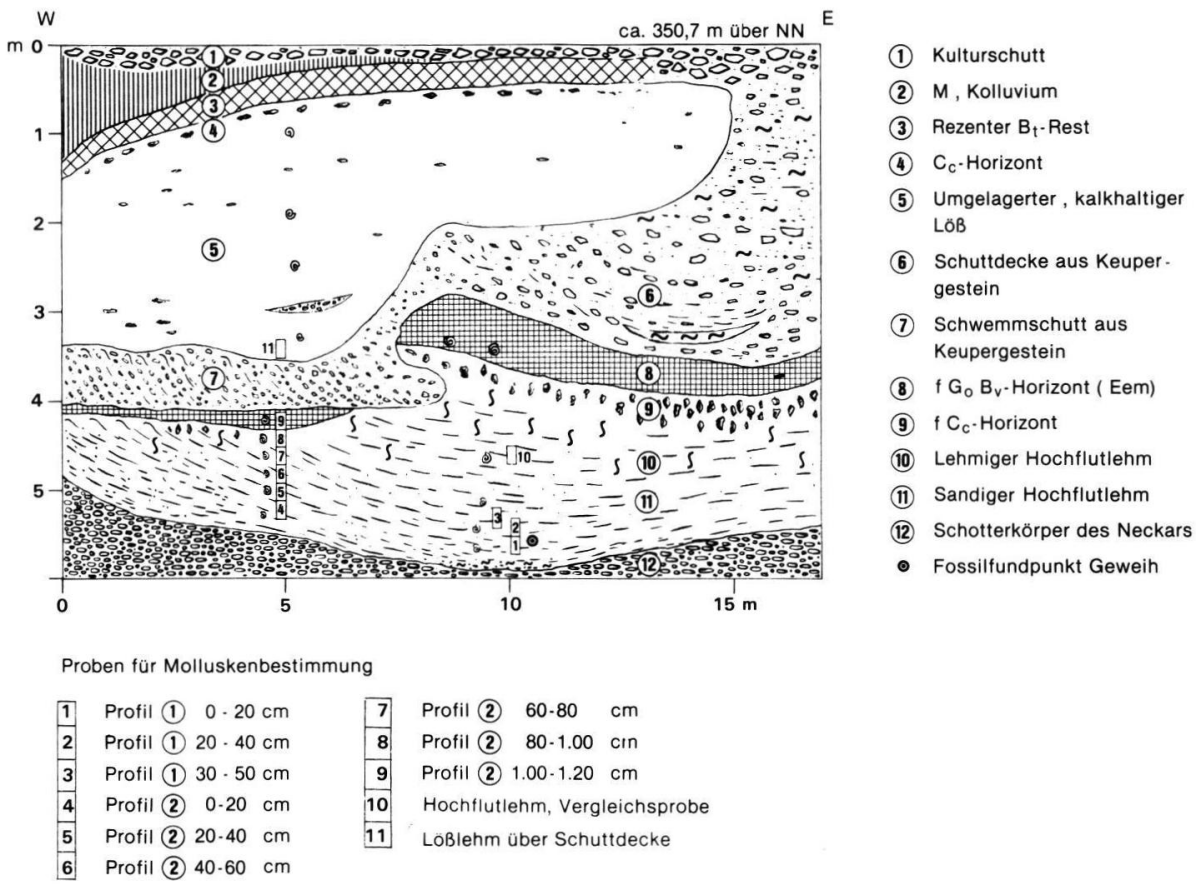

Abb. 3: Aufnahme des Straßenbau-Aufschlusses von Rottenburg mit Lage der untersuchten Molluskenproben.

Gleys in einem zeitlich begrenzten Abschnitt einer Warmzeit rekonstruieren. Da es zu einer starken Verbraunung und vollkommenen Entkalkung gekommen ist, handelte es sich mit Sicherheit nicht um die Endphase eines Interglazials. Es liegt vielmehr nahe, den Hochflutlehm als Ablagerung im frühen bis mittleren Abschnitt eines Interglazials zu deuten. Gegen eine solche Interpretation würde auch die im Hochflutlehm eingebettete Geweihstange nicht sprechen.

Über dem Auensediment folgte im westlichen Teil des Aufschlusses eine Mergelfließerde aus violetten bis grauen Gesteinsbruchstücken des Gipskeupers, die in einer folgenden Kaltzeit die fluviatilen Sedimente vom höheren Hang her überfahren hat (Abb. 3, Schicht 6). Die Schuttdecke wies Kryoturbationen auf und verzahnte sich mit einem kalkhaltigen steinigen Fließlöß. Auch die leichte Aufpressung des Hochflutlehms im mittleren Abschnitt des Aufschlusses dürfte auf periglaziale Einwirkungen in der nachfolgenden Kaltzeit zurückzuführen sein. Vor Ablagerung der Mergelfließerde und des verlagerten Lösses, die aufgrund der Verzahnung altersgleich sind, erfolgte lokal eine deutliche fluviatile Überprägung des Hanges. So wurde im mittleren Abschnitt des Aufschlusses der $\mathrm{GoB}_{\mathrm{v}}$-Horizont des Braunerde-Gleys in einem flachen Gerinnebett ausgeräumt. Die Füllung der Hohlform (7) bestand aus einem dachziegelartig gelagerten Schwemmschutt mit sehr vielen aufgearbeiteten
Mergel- und Tonsteinbröckchen des Gipskeupers. In durchgeführten Schotteranalysen konnten vereinzelt auch gröbere, gut gerundete Buntsandstein- und Muschelkalkgerölle nachgewiesen werden. Trotz dieser Fremdbeimengung handelte es sich mit Sicherheit nicht um eine Neckarablagerung, sondern um das Sediment einer Hangrinne, in der neben Lokalmaterial wohl auch Neckargerölle von höheren Terrassen aufgearbeitet wurden. Die Erosionsform zeigt an, daß durchaus mit einer Kappung des Auenbodens, vermutlich auch außerhalb der verfüllten Mulde, gerechnet werden muß.

Der abschließende Fließlöß (5) wies vereinzelt eingelagerte Gerölle, Steine und Schwemmschuttstreifen auf. Eine Untergliederung durch fossile Bodenhorizonte war leider nicht vorhanden. In dem kalkhaltigen Sediment ließen sich allerdings Lößschnecken nachweisen, die in die paläontologischen Untersuchungen mit einbezogen wurden.

Als rezenter Boden trat auf dem Löß der humosbraune $B_{t}$-Rest einer Parabraunerde (3) mit einer starken Ton-Humus-Durchschlämmung auf, wie sie auch für das angrenzende Korngäu als typisch erkannt worden ist (vgl. BiBUS 1985). Eine starke anthropogene Überprägung des rezenten Bodens zeigte sich in einer bis $1,50 \mathrm{~m}$ mächtigen kolluvialen Überdeckung (2) am Rande einer angeschnittenen Hohlform. 
Den Abschluß des Profiles bildete die Befestigung aus Schutt und Schottern (1) im Bereich einer alten Römerstraße.

Nach der Höhenlage der Neckarsedimente über der Talaue, mehr jedoch aufgrund der periglazialen Deckschichten und des Ausbildungsgrades des fossilen Auenbodens muß davon ausgegangen werden, daß es sich bei dem fluviatilen Komplex um eine rißzeitliche Neckarterrasse mit dem letztinterglazialen Hochflutlehm und Relikten eines eeminterglazialen Bodens handelt. Aus dem Bereich des Hochflutlehmes wurde ein Standardprofil (Probe 4-10) unter Berücksichtigung von Vergleichsproben malakozoologisch untersucht (vgl. Kap. 4).

\section{Die Straßenbau-Aufschlüsse am S-Rand von Heilbronn-Böckingen}

Durch Straßenbaumaßnahmen wurden am südlichen Ortseingang von Heilbronn-Böckingen im Jahre 1983 große Aufschlüsse am Westhang des Neckartales geschaffen, die z. T. bis in die liegenden Keupergesteine reichten. Außerdem war unterhalb der Baustelle im Herbst 1984 durch Gasleitungsgräben die Neckaraue oberflächennah aufgeschlossen, so daß ein durch- gängiges Längsprofil von der Aue in den unteren Talhangbereich erstellt werden konnte (vgl. Abb. 4).

Morphologisch tritt über der kaum gegliederten Talaue eine ca. $12 \mathrm{~m}$ hohe Terrassenstufe auf, an deren Fuß die Eisenbahnlinie nach Bietigheim verläuft. Im Bereich der Aue liegen braune Auenböden aus kalkhaltigen schluffigen Lehmen (maximal 1,60 m) vor, die von grauen kalkhaltigen Hochflutsanden unterlagert werden. Ab 2,10 bis 2,50 m u. Fl. folgen die sandigen Kiese der Talauenfüllung.

Die untere Talhangstufe wird von einem Neckarschotterkörper mit abschließendem Hochflutlehm und einer mehrgliedrigen Lößdeckschicht aufgebaut.

Die 4 m mächtigen, sandigen Kiese (12) überlagerten in der Baugrube an der Bahnunterführung (R 351392 $\mathrm{H}$ 544336) anstehenden Kalk (vgl. Abb. 5, Schicht 13). Neben Muschelkalkgeröllen ließen sich auch viele aus Buntsandstein nachweisen. Nahe der Ober- und Untergrenze des Schotterkörpers waren zahlreiche grobe Gesteinsplatten eingelagert, die eine Aufarbeitung von periglazialem Schutt zur Ablagerungszeit im Einzugsgebiet anzeigten. Der hangende Hochflutlehm (Abb. 5, 11) war rötlichbraun gefärbt und von einzelnen Geröllen sowie von Kies- und Sandlagen durchsetzt. In dem kalkhaltigen Hochflutsediment

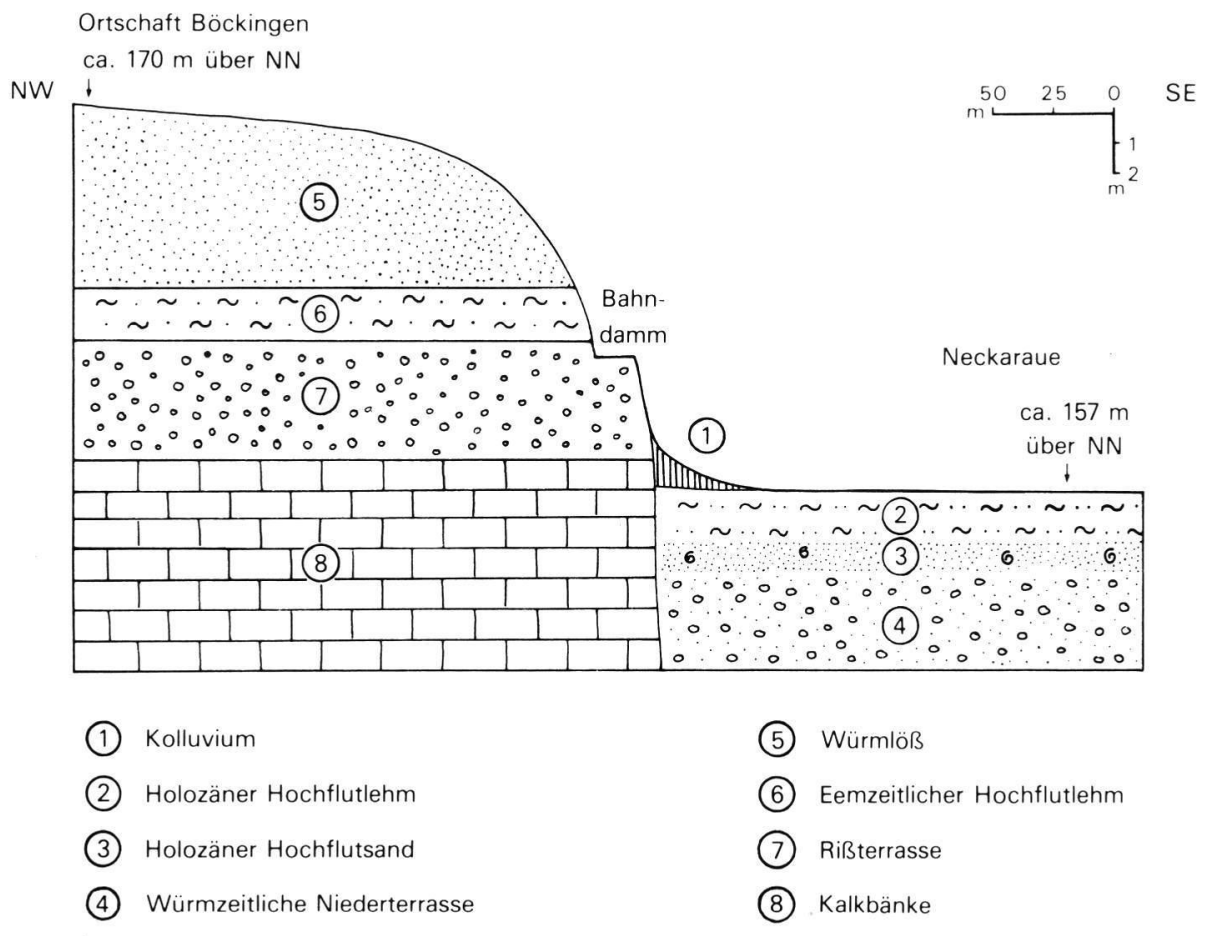

Abb. 4: Schichtabfolge im talauennahen Hangbereich am südlichen Ortsrand von Heilbronn-Böckingen. 


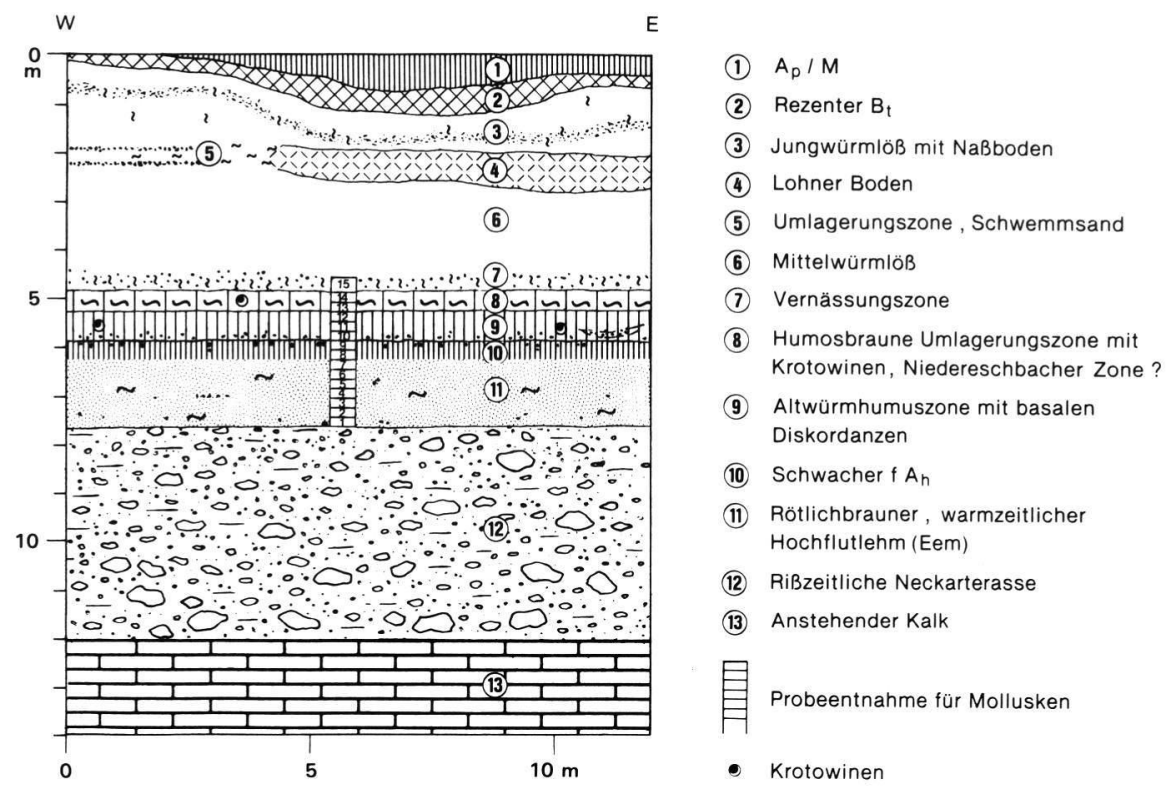

Abb. 5: Ausschnitt aus dem Straßenbau-Aufschluß Heilbronn-Böckingen mit Lage der untersuchten Molluskenproben.

traten viele große Schnecken und deren Bruchstücke auf. Nach durchgeführten Korngrößenanalysen handelte es sich um einen tonigen Lehm, der in unterschiedlicher Höhe eine nahezu identische Korngrößenzusammensetzung besaß (vgl. Abb. 6). Eine Verwitterung von der ehemaligen Oberfläche her ließ sich somit nicht nachweisen. Eine starke postsedimentäre pedogene Überprägung kann auch deshalb ausgeschlossen werden, da die vielen eingelagerten Schnecken einen primären Kalkgehalt anzeigten. Die rötlichbraune Färbung und der hohe Tongehalt muß auf die Abtragung von verbraunten Böden und Keupermergeln zur Ablagerungszeit zurückgeführt werden. Es handelte sich somit zweifelsfrei um ein Sediment aus Bodenmaterial vom Typ des braunen Auenbodens, welches den Schluß zuläßt, daß zur Sedimentationszeit des Hochflutlehms im Einzugsgebiet weiträumig verbraunte Böden entstanden. Die Ablagerung dürfte deshalb, im Gegensatz zu den Verhältnissen in Rottenburg, nicht am Anfang, sondern eher zur Mitte oder gegen das Ende eines Interglazials erfolgt sein. Als autochthone Bodenmerkmale wies der Hochflutlehm nur einen geringmächtigen, schwachen $A_{\mathrm{h}}$-Horizont (10) auf. Eine an der Obergrenze ausgebildete Kieslage zeigte außerdem eine deutliche Diskordanz zu den hangenden Lößdeckschichten an.

Das Lößprofil war durch mehrere Bodenhorizonte von unterschiedlicher Ausprägung gegliedert. Im Vergleich mit dem gleichzeitig aufgenommenen Lößprofil der ehem. Ziegelei Böckingen ließen sich die
Böden eindeutig in die Würmlößgliederung einordnen, wie sie z. B. von SEMmEL (1968) für Hessen aufgestellt worden ist.

Im untersten Abschnitt lag eine Altwürm-Humuszone (9) vom Typ der Mosbacher Humuszonen vor. Neben aufgearbeiteten Kiesen konnten sehr häufig Krotowinen beobachtet werden. Bei dem bereits erwähnten schwachen $A_{h}$-Horizont auf dem Hochflutlehm (10) handelte es sich vermutlich um den untersten Abschnitt der Humuszone. Auf jeden Fall war die Humuszone aufgrund der liegenden Diskordanz sowie ihrer Ausbildung auf lößhaltigem Ausgangssubstrat jünger als der Hochflutlehm. Über der Humuszone folgte eine graurötlichbraune Umlagerungszone (8) aus verbrauntem und humosem Bodenmaterial, welches in hessischen Profilen als Niedereschbacher Zone bezeichnet wird (SEMMEL 1969). Auch in dieser Schicht waren mit gelbem Löß gefüllte Krotowinen vorhanden. Ein Vergleich mit dem Profil der ehem. Ziegelei Böckingen zeigte, daß dort die gleiche Abfolge über dem Rest der letztinterglazialen Parabraunerde auftritt. Für diesen Profilabschnitt hat neuerdings BLEICH in Baden-Württemberg (BLEICH \& WURSTER 1983; BLEICH et al. 1984) aufgearbeitete vulkanische Gläser in mehreren Aufschlüssen nachgewiesen, die wohl mit dem in gleicher stratigraphischer Position liegenden Älteren Wehrer Bims (FRECHEN 1975: 176) bzw. dem Metternicher Tuff (LÖHR \& BRUNNACKER 1974) am unteren und mittleren Mittelrhein parallelisiert werden können (vgl. auch BIBUS 1980: 183). 


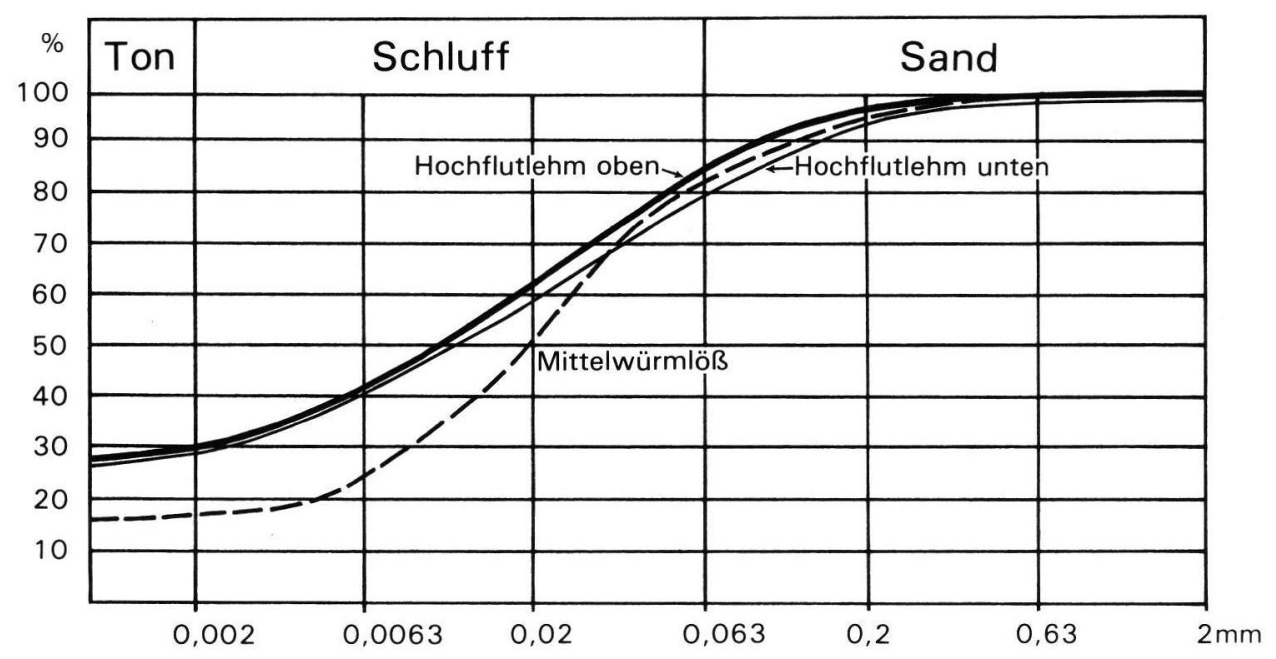

Abb. 6: Korngrößenzusammensetzung des eemzeitlichen Hochflutlehms und des Wurmlösses in Heilbronn-Böckingen.

Über dem basalen Altwürmabschnitt und der Niedereschbacher Zone folgte ein braungelber Löß mit einer basalen Vernässungszone (Abb. 5, Schicht 7), der durch einen schwachen bräunlichen Boden zweigeteilt wurde. Nach den Profilmerkmalen handelte es sich um den Lohner Boden (4), der auch in der Ziegelei Böckingen aufgeschlossen ist. Der zwischen der Niedereschbacher Zone und dem Lohner Boden liegende Löß umfaßte deshalb zeitlich das Mittelwürm. Innerhalb des geringmächtigen Jungwürmlösses trat noch ein schwacher Naßboden (3) der Erbenheimer Böden auf, der allerdings nicht sicher eingeordnet werden konnte. Abgeschlossen wurde das Profil von der stark erodierten, rezenten Parabraunerde, die in Muldenlage eine kolluviale Überdeckung aufwies.

Wenngleich die Deckschichten der Terrasse nicht alle Würmböden enthalten, so kann mit den vorhandenen Horizonten eindeutig bewiesen werden, daß es sich bei dem liegenden Hochflutlehm um eine letztinterglaziale Bildung und bei dem Neckarschotterkörper um eine rißzeitliche Terrasse handelte. Für die Molluskenbestimmungen wurde der gesamte Hochflutlehm und die Lößdeckschichten bis in den Mittelwürmlöß beprobt.

\section{Die Molluskenfaunen}

Unser Wissen von den letztinterglazialen Molluskenfaunen des Neckargebietes basiert im wesentlichen auf den Untersuchungen der Kalktuffe von Dettingen und Dießen bei Horb (GEYER 1912 u. 1913; MAECK 1963; MÄGDEFRAU \& MAECK 1965) und der Seelberg-
Ablagerungen von Stuttgart-Bad Cannstatt (GEYER 1913; KRANZ, BERCKHEIMER \& WÄGELE 1930; MÜNZING 1966, 1973). Mit den Straßenbau-Aufschlüssen bei Rottenburg und Heilbronn-Böckingen liegen jetzt zwei weitere, molluskenführende Quartärprofile aus jener Zeit vor. Die besondere Bedeutung dieser neuen Fundstellen liegt darin, daß sich ihre Faunen bestimmten Zeitabschnitten des letzten Interglazials zuordnen lassen. In Heilbronn-Böckingen waren auch die basalen Abschnitte des würmzeitlichen Lößprofils Gegenstand der malakazoologischen Untersuchungen.

Für die Erfassung der Molluskenbestände wurden den Profilen $20 \mathrm{~cm}$ mächtige und genau eingemessene Sedimentproben entnommen (Abb. 3 u. 5). Das Auslesen und Bestimmen der Molluskenreste erfolgte unter dem Stereomikroskop im Anschluß an das Schlämmen der Proben. Aus den Schalenresten wurde, entsprechend den Angaben bei LOŽEK (1964), näherungsweise die Stückzahl der einzelnen Arten ermittelt. In den Artenlisten (Tabelle 1 u. 2) und Molluskenspektren (Abb. 7) werden die Arten in Anlehnung an LOŽEK (1964) und EvANS (1972) vier verschiedenen ökologischen Gruppen zugeordnet. In den Tabellen finden sich außerdem Hinweise auf die biostratigraphische Bedeutung einzelner Arten.

Fossile Molluskenfaunen aus fluviatilen Ablagerungen sind zusammengeschwemmte Bestände, die zum einen aus der Talaue selbst und zum anderen von den angrenzenden Talhängen stammen. Sie ermöglichen im allgemeinen einen guten Überblick über die malakologischen Verhältnisse im Einzugsgebiet des 


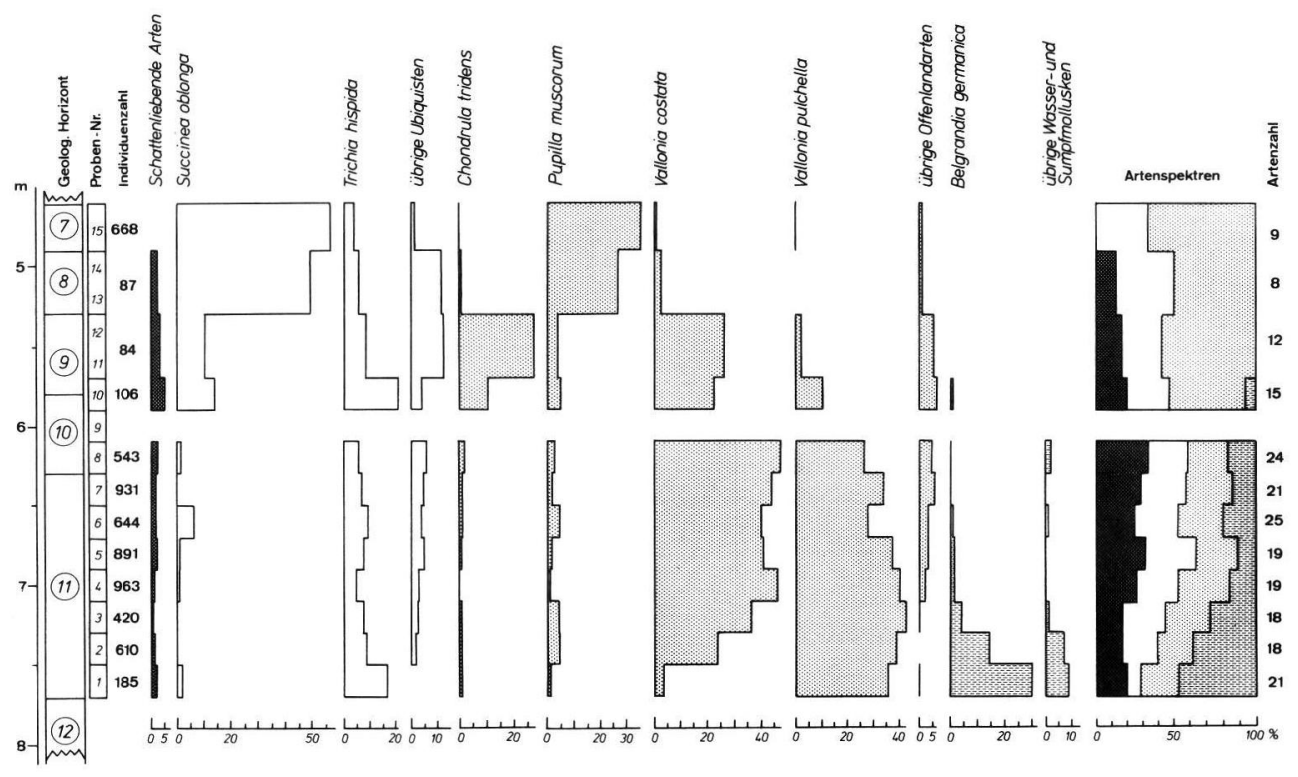

Abb. 7: Struktur der eem- und altwürmzeitlichen Molluskenfaunen der Straßenbau-Aufschlüsse am Südrand von Heilbronn-Böckingen.

jeweiligen Gewässers (LOŽEK 1976), doch muß die Interpretation der Befunde mit der nötigen Vorsicht erfolgen. Von den Hochfluten werden in erster Linie die Bewohner der Talaue und die Bewohner der offenen Landschaft erfaßt. Die Schnecken der Hangwälder gelangen dagegen nur sporadisch in den Einzugsbereich des fließenden Wassers und sind in solchen Ablagerungen entsprechend selten anzutreffen. Darüber hinaus findet durch die sortierende Wirkung des fließenden Wassers eine Auslese zu Gunsten kleiner und kleinster Formen statt. Größere Molluskengehäuse sind eher unterrepräsentiert, weil sie beim Transport leicht zerstört werden oder im Wasser rasch absinken (GEYER 1914, 1923; 1927; LOŽEK 1964).

So setzen sich auch die Molluskenbestände der Hochflutlehme von Rottenburg und Heilbronn-Böckingen vorwiegend aus Formen zusammen, deren Lebensraum die Flußaue selbst gewesen ist (Tabelle 1, 1-10; Tabelle 2, 1-8). Hierher gehören zunächst Bewohner von Gewässern aller Art (Bithynia tentaculata, Radix peregra, Bathyomphalus contortus, Pisidium milium, Pisidium subtruncatum und Pisidium casertanum), Bewohner von temporären Kleingewässern (Valvata cristata, Galba truncatula, Anisus leucostomus, Pisidium obtusale), Formen aus Tümpeln und Altwässern (Planorbis planorbis, Hippeutis complanatus, Gyraulus crista) und Arten des fließenden Wassers (Theodoxus fluviatilis, Valvata piscinalis, Belgrandia germanica, Ancylus fluviatilis, Unio crassus, Pisidium amnicum und Pisidium nitidum). Dazu kommen ufer- und sumpfbewohnende Landschnecken (Carychium minimum, Cochlicopa nitens, große Vertreter der Succineidae, Vertigo antivertigo und Zonitoides nitidus). Unter den Landschnecken haben feuchtigkeitsliebende, sonst aber eher anspruchslose Ubiquisten (Carychium tridentatum, Cochlicopa lubrica, Succinea oblonga, Punctum pygmaeum, Nesovitrea hammonis, Trichia hispida und Nacktschnecken) einen erheblichen Anteil. Von den schattenliebenden Arten (Waldschnecken i. w. S.) finden sich vor allem Auwaldbewohner wie Aegopinella spec., Clausilia pumila, Bradybaena fruticum, Trichia villosa oder Arianta arbustorum. Anspruchsvollere Waldarten, welche die feuchte und kühle Flußniederung eher meiden (Orcula doliolum, Ena montana, Cochlodina spec., Ruthenica filograna, Macrogastra plicatula, Helicodonta obvoluta), sind selten. Am stärksten vertreten sind naturgemäß die Offenlandarten, da sie am leichtesten vom sammelnden Wasser weggetragen werden können. Besonders dominant sind einige kleine und kleinste Formen aus dieser Gruppe (Vertigo pygmaea, Pupilla muscorum, Vallonia costata und Vallonia pulchella), von welchen ein großer Teil ebenfalls aus der Talaue stammen dürfte. Dagegen sind die Standorte der übrigen Vertreter aus dieser Gruppe (Truncatellina cylindrica, Granaria frumentum, Chondrula tridens, Helicopsis striata) eher an xerotherm geprägten, grasigen oder felsigen Talhängen zu suchen. Gleiches gilt für einige subthermophile Ubiquisten wie Abida secale und Clausilia parvula. 
Die meisten der in den Hochflutlehmen von Rottenburg und Heilbronn-Böckingen vorkommenden Molluskenarten sind feste Bestandteile sowohl der interglazialen als auch der postglazialen Fauna des Neckargebietes. Einzelne Arten jedoch haben die Siedlungsgebiete, die sie in den pleistozänen Warmzeiten innehatten, im Postglazial nicht wiedergewinnen können. Solche Formen sind als interglaziale Leitarten biostratigraphisch besonders bedeutsam. An erster Stelle ist hier Belgrandia germanica zu nennen, die in Rottenburg und Böckingen gleichermaßen stark vertreten ist.

Die Belgrandien, welche heutzutage ein zersprengtes Areal im nordwestlichen Mittelmeerraum besiedeln, waren in pleistozänen Warmzeiten über weite Teile West- und Mitteleuropas verbreitet (STEUSLOFF 1953). Auch in Baden-Württemberg sind sie aus zahlreichen Interglazialbildungen unterschiedlichen Alters bekannt (DEHM 1951; GEYER 1912, 1913, 1914, 1915; MÜNZING 1966). Im Riß/Würm-Interglazial (Eem) traten sie bei uns zum letzten Mal auf.

Auch Ruthenica filograna und Clausilia pumila ${ }^{1}$ ) können im Neckargebiet als interglaziale Leitarten gelten.

Das Verbreitungszentrum dieser Arten liegt im östlichen Europa. In verschiedenen pleistozänen Warmzeiten sind Ruthenica filograna und Clausilia pumila weit nach W vorgestoßen (GEYER 1927; EVANS 1972) und sind dort von zahlreichen Fundorten bekannt, die außerhalb ihrer postglazialen Verbreitungsgebiete liegen, so auch aus dem Gebiet des oberen und mittleren Neckars (FRAAS 1896; GEYER 1912, 1913, 1914, 1915, 1920; KRANZ, BERCKHEMER \& WÄGELE 1930; MƯNZING 1966, 1968 u. 1973). Für Ruthenica filograna markieren heute einige isolierte Vorposten auf der mittleren Schwäbischen Alb die Westgrenze des Verbreitungsgebietes. Clausilia pumila erreichte im Postglazial Baden-Württemberg nicht mehr.

\section{Die Mollusken der Straßenbau-Aufschlüsse von Rottenburg}

Die Hochflutlehme von Rottenburg führen interglaziale Molluskenfaunen mit zahlreichen wärmeliebenden Elementen (Tabelle 1). Leitart ist Belgrandia germanica, welche in allen Proben mehr oder weniger zahlreich vertreten ist. Die stratigraphischen Verhältnisse (Kapitel 2) sprechen für eine Datierung der Ablagerungen in das Riß/Würm-Interglazial.

1) Clausilia pumila ist im Gegensatz zu Ruthenica filograna kein warmzeitliches Faunenelement, sondern eine klimatisch indifferente Art. Im östlichen Europa tritt sie auch in feuchteren Abschnitten der Kaltzeiten auf (LOŽEK 1964).
Die Molluskenbestände der Proben 1-9, die von der Basis und vom Randbereich einer rinnenartigen Vertiefung im rißzeitlichen Schotterkörper stammen (Abb. 3), stimmen in ihrer Zusammensetzung weitgehend miteinander überein. Dagegen weist eine im Zentrum und nahe der Obergrenze der mit Hochflutsedimenten ausgefüllten Vertiefung entnommene Probe (Probe 10, Abb. 3) einige bemerkenswerte Unterschiede auf. Leider lag aus diesem interessanten Profilabschnitt, bei dem es sich vermutlich um eine jüngere Verfüllung èiner Depression in bereits abgelagerten, älteren Sedimenten handelt, nur diese einzige Probe vor, welche zudem nur relativ wenige Mollusken enthielt. Eine nachträgliche Entnahme von Material ließen die rasch fortschreitenden Bauarbeiten nicht $\mathrm{zu}$.

Die Proben 1-9 führen klassische Discus ruderatusFaunen. Derartige Faunen sind für frühe Abschnitte von Warmzeiten sehr charakteristisch. In der Molluskenfauna aus Probe 10 hingegen ist die frühwarmzeitliche Leitart Discus ruderatus durch Discus rotundatus ersetzt. Die Fauna weist außerdem eine ganz andere Artenzusammensetzung und einen höheren Anteil an schattenliebenden Formen (Waldarten i. w. S.) auf (Tabelle 1).

Alle bisherigen Befunde (LOŽEK 1969, 1976) weisen darauf hin, daß die Malakofauna am Beginn des letzten Interglazials der altholozänen Fauna sehr ähnlich und weitgehend den gleichen Wandlungen unterworfen war wie im Postglazial. Im Postglazial Mitteleuropas ist der Rückzug der altholozänen Leitart Discus ruderatus an der Wende Boreal/Atlantikum ein besonders markantes Ereignis. Discus ruderatus eine kontinentale Waldschnecke - wird in dieser Zeit mehr und mehr von ihrer westeuropäischen Schwesterart, Discus rotundatus, verdrängt - ein Phänomen, das mit dem Einsetzen von feuchterem, ozeanisch geprägtem Klima einhergehen dürfte (DEHM 1967; RÄHLE 1983).

In den Hochflutlehmen von Rottenburg ist ein ganz entsprechendes, faunengeschichtliches Ereignis zu beobachten. Die Discus ruderatus-Faunen (Proben 1-9) wären somit und in Analogie zu den Verhältnissen im Postglazial in einen früheren, die Fauna ohne Discus ruderatus dagegen in einen späteren (möglicherweise mittleren) Abschnitt des Eem-Interglazials zu stellen. Diese Schlußfolgerung steht ganz im Einklang mit den Ergebnissen der parallel durchgeführten, pedologischen Untersuchungen (Kapitel 2).

Die Hochflutlehme von Rottenburg, insbesondere diejenigen, welche Discus-ruderatus-Faunen führen, sind mit großer Wahrscheinlichkeit älter als die hochinterglazialen Kalktuffe von Dettingen und Dießen 
bei Horb, von denen lediglich Faunen mit Discus rotundatus, nicht aber solche mit Discus ruderatus bekannt sind (MAECK 1963).

Der im Hangenden aufgeschlossene, verlagerte Löß (Horizont 5, Abb. 3) enthielt an seiner Basis (Probe 11, Tabelle 1) eine hochglaziale Fauna der feuchten Lößlandschaft, die nach den Dominanzverhältnissen als „Succinea oblonga-Fauna mit Pupillen und Columella columella" (MÜNZING 1971) bezeichnet werden kann.

\section{Die Mollusken der Straßenbau-Aufschlüsse von Heilbronn-Böckingen}

Die Mollusken des Profils stammen aus einem $310 \mathrm{~cm}$ mächtigen Schichtpaket, welches von der Basis der Hochflutlehme bis in eine Vernässungszone an der Basis der mittelwürmzeitlichen Lösse reicht (Abb. 5). Mit Hilfe der Molluskenbestände läßt sich dieser Sedimentkomplex in wenigstens drei Abschnitte gliedern:

Aus den Hochflutlehmen (Tabelle 2, 1-9/10; Abb. 7) liegt eine relativ artenreiche Molluskenfauna mit einer ganzen Anzahl warmzeitlicher Elemente vor. Darunter befinden sich als interglaziale Leitarten Belgrandia germanica, Ruthenica filograna, Clausilia pumila und - als Leitart für das Eem-Interglazial Theodoxus fluviatilis (GEYER 1914; JAECKEL 1962; STEUSLOFF 1953).

Besonders bezeichnend für diese Fauna ist der hohe Anteil der Offenlandarten (darunter die kontinentale Steppenart Chondrula tridens) sowie die drastische Abnahme der Individuenzahlen bei den thermophilen Belgrandien (Tabelle 2). Unter Berücksichtigung der paläopedologisch-sedimentologischen Befunde (Kapitel 3) läßt sich daraus der Schluß ziehen, daß hier eine bereits abklingende Phase des letzten Interglazials dokumentiert ist. Die Hochflutsedimente von Heilbronn-Böckingen dürften dabei älter sein als die spätinterglazialen „Neckarauemergel” von der Deckerstraße in Stuttgart-Bad Cannstatt (MÜNZING 1973: Tabelle 3, I/II), wo die Molluskenfaunen einen ähnlich hohen Anteil an Offenlandarten, aber erheblich weniger warmzeitliche Formen aufweisen, und jünger als die „Neckarauemergel” von der HeinrichEbner-Straße (früher Ganzhornstraße), ebenfalls in Stuttgart-Bad Cannstatt, wo Steppenelemente noch keine Rolle spielen (KRANZ et al. 1930; MÜNZING 1966, 1973).

Mit der Altwürmhumuszone (Horizont 9, Abb. 5) beginnt ein zweiter, im Sedimentkomplex von Heilbronn-Böckingen malakozoologisch faßbarer $\mathrm{Ab}$ schnitt. Eine Diskordanz an ihrer Basis stellt allerdings eine Lücke in der faunistischen Überlieferung dar. Im Liegenden der Altwürmhumuszone (Basis Probe 10) finden sich - über einer fundleeren Kieslage (Probe 9, Abb. 7) - noch Reste der warmzeitlichen Fauna (Belgrandia germanica, Ruthenica filograna). Länger hält sich die klimatisch widerstandsfähige Clausilia pumila (Tabelle 2). Im übrigen führt die Altwürmhumuszone (Proben 10-12, Abb. 7) eine typische, frühkaltzeitliche Chlondrula-tridensFauna (LOŽEK 1964). Es handelt sich dabei um eine Molluskengesellschaft der offenen, steppenartigen Landschaft, die weder ausgesprochen warmzeitliche, noch ausgesprochen kaltzeitliche Faunenelemente enthält. In Heilbronn-Böckingen ist sie noch verhältnismäßig artenreich und führt neben genügsamen Arten offener Flächen (Pupilla muscorum, Vallonia costata, Vallonia pulchella, Helicopsis striata) auch anspruchsvollere Formen wie Truncatellina cylindrica, Vertigo pygmaea und Granaria frumentum. Die faunistischen Befunde stehen also ganz im Einklang mit dem Nachweis eines interstadialen Bodenhorizontes in diesem Abschnitt des Profils.

Die vorliegende Fauna hat einen ganz anderen Charakter als die „kalten” Steppenfaunen vom Helicopsis striata-Typus, welche MÜNZING (1971) aus einem, ebenfalls als Altwürmhumuszone bezeichneten Abschnitt des Lößprofils der Lehmgrube in den nahegelegenen Ziegelwerken von Heilbronn-Böckingen beschrieben hat. Faunen mit Chondrula tridens, die sich von denjenigen der neuen Aufschlüsse bei Böckingen nur unwesentlich unterscheiden, sind aus Travertinsanden der Seelberg-Ablagerungen von Stuttgart-Bad Cannstatt bekannt (KRANZ et al. 1930; MÜNZING 1966, 1973).

Der folgenden Umlagerungszone (Niedereschbacher Zone ?, Horizont 8, Abb. 5) fehlen alle anspruchsvolleren Molluskenarten (Tabelle 2). In ihren oberen Partien (eine Verunreinigung durch Material aus Krotowinen ist nicht auszuschließen) deutet sich bereits der Übergang zu einer Lößfauna an, wie sie aus einer ca. $30 \mathrm{~cm}$ mächtigen Vernässungszone an der Basis der mittelwürmzeitlichen Lösse vorliegt (Probe 15, Abb. 7). Es handelt sich hier um eine Fauna der feuchten Lößlandschaft mit Succinea oblonga als dominierender Art, Pupilla muscorum und Columella columella als hochglazialem Faunenelement. Trichia hispida spielt lediglich eine untergeordnete Rolle. Faunen dieses Typs sind nach MÜNZING (1971) im mittleren Neckarraum für das Mittelwürm charakteristisch. Dagegen verweist der Fund eines einzelnen Exemplares von Vallonia pulchella nach den Ausführungen von REMY (1968) über das Auftreten dieser Art in der letzten Kaltzeit noch ins Altwürm. 


\section{Zusammenfassende Schlußbetrachtung}

Aus dem Neckartal zwischen Rottenburg und Heilbronn werden von zwei Stellen ca. 5-6 m (Oberkante) über der Talaue liegende Terrassen mit einem abschließenden Hochflutlehm und kaltzeitlichen Deckschichten beschrieben, die nach ihrer geomorphologischen Position als würmzeitliche Niederterrasse gedeutet werden könnten. SCHMIDT (1932: 29) sieht ein von der Höhenlage her vergleichbares Terrassensediment am „Hohen Rain” bei Rottenburg sogar als römerzeitliche Ablagerung an. Die Analyse der Deckschichten und Paläoböden sowie die Bestimmung der Mollusken hat jedoch für die hier näher beschriebenen Lokalitäten gezeigt, daß es sich in beiden Fällen um rißzeitliche Schotterterrassen mit eeminterglazialen Hochflutlehmen und abschließenden würmzeitlichen Decksedimenten handelt. Der unterschiedliche Ausbildungsgrad der eemzeitlichen Böden und Hochflutlehme ließ weiterhin den Schluß zu, daß der Hochflutlehm bei Rottenburg in einem früheren (bis möglicherweise mittleren) und der Hochflutlehm von Heilbronn-Böckingen in einem späteren $A b$ schnitt des Eem-Interglazials zur Ablagerung gelangte. Diese Ansicht konnte durch die Molluskenfunde voll bestätigt werden.

Die Hochflutlehme von Rottenburg erwiesen sich insgesamt älter als die letztinterglazialen Kalktuffe von Dettingen und Dießen bei Horb und älter als die in das Eem zu stellenden „Neckaraumergel” von Stuttgart-Bad Cannstatt. Die Hochflutlehme von Böckingen nehmen dagegen zeitlich eine Mittelstellung zwischen älteren und jüngeren eemzeitlichen Hochflutsedimenten von Stuttgart-Bad Cannstatt ein.

Die malakozoologischen Untersuchungen lieferten eine Anzahl neuer und ergänzender Daten zur $\mathrm{Ge}$ schichte der südwestdeutschen Molluskenfauna im Eem-Interglazial. Besonders hervorzuheben sind der Nachweis einer alteemzeitlichen Discus ruderatusFauna (Rottenburg) und die Möglichkeit einer zeitlichen Korrelation der Böckinger Funde mit Molluskenfaunen aus verschiedenen Schichten der SeelbergAblagerungen von Stuttgart-Bad Cannstatt.

Die vorgelegten Befunde sind außerdem für die Rekonstruktion der jungpleistozänen Talentwicklung von Bedeutung, da mit den „Auemergeln" von Stuttgart-Bad Cannstatt und Untertürkheim (REIFF 1965: 118; 1973: $410 \mathrm{f}$.) inzwischen mehrere letztinterglaziale Hochflutlehme in verschiedenen Abschnitten des Neckartales nachgewiesen sind, die in gleicher geomorphologischer Position über der heutigen Talaue liegen. Auch ACHILlES (1939: 60) gibt im mittleren Neckartal für seine jüngste Rißterrasse einen Auenabstand von $6 \mathrm{~m}$ an. Zudem wurde ein in situ befindlicher letztinterglazialer Parabraunerderest in einem Nebentälchen des Neckars westlich von Tübingen fast im Niveau der Tälchensohle und insgesamt nur $8 \mathrm{~m}$ über der Neckaraue beschrieben (BIBUS 1985: 117). Dies alles sind im Neckartal Hinweise für eine nur geringe Höhenlage der Eemablagerungen und der Rißterrasse über der holozänen Talsohle. Allerdings ist im Einzelfall nicht abzuschätzen, in welchem Maße junge Tektonik berücksichtigt werden muß (Rottenburger Flexur, Beckenlage von Bad Cannstatt und Heilbronn-Böckingen). Der identische Auenabstand der verschiedenen Hochflutlehme spricht eigentlich gegen jungpleistozäne Verstellungen (vgl. auch MÜHLFELD 1953: 48 ff.; REIFF 1965: 124), da gleiche Verstellungsbeträge an den verschiedenen Lokalitäten unwahrscheinlich sind. Mit Sicherheit kann aber nach den vorgelegten Ergebnissen ein Eintiefungsbetrag des Neckars bei Rottenburg von 70 m (MADER 1978) seit dem Mindel/Riß-Interglazial ausgeschlossen werden.

\section{Schriftenverzeichnis}

ACHILles, K. A. (1939): Diluvialgeologische Untersuchungen im mittleren Neckartal. - Jh. Ver. vaterl. Naturkde. Württ., 95: 1-110, 10 Phot., 6 Abb., 26 Tab., 4 Taf.; Stuttgart.

BIBUS, E. (1974): Abtragungs- und Bodenbildungsphasen im Rißlöß. - Eiszeitalter u. Gegenwart, 25: 166-182, $6 \mathrm{Abb}$.; Öhringen (Württ.).

- (1980): Zur Relief-, Boden- und Sedimententwicklung am unteren Mittelrhein. - Frankf. geowiss. Arb., D, 1: 296 S., 50 Abb., 8 Tab.; Frankfurt a. M.

- (1985): Zur jungen Relief- und Bodenentwicklung in der Umgebung von Tübingen. - Z. Geomorph., N. F., Suppl.-Bd. 56: 109-124, 2 Phot., 4 Abb., 2 Tab.; Berlin-Stuttgart.

Bleich, K. E., HÄDrich, F. \& WurSTER, R. (1984): Die Bedeutung vulkanischer Glasfunde für die Chronostratigraphie des oberrheinischen Lösses. - Ber. naturforsch. Ges. Freiburg i. Br., 74: 5-24, 5 Abb., 2 Tab.; Freiburg.

- \& WURSTER, R. (1983): Eine Lage von vulkanischen Gläsern im süddeutschen Jungpleistozän. - Mitt. dt. bodenkundl. Ges., 38: 461—466, 5 Abb.; Göttingen.

Вӧнм, Е. (1927): Das östliche Vorland des mittleren Schwarzwalds. Seine morphologische Entwicklung und deren geologische Grundlagen. - Jh. Ver. vaterl. Naturkde. Württ., 83: 59-104; Stuttgart.

DeHM, R. (1951): Mitteldiluviale Kalktuffe und ihre Molluskenfauna bei Schmiechen nahe Blaubeuren. - N. Jb. Geol. Paläont., Abh., 93: 247-276, 2 Abb., 2 Taf.; Stuttgart.

- (1967): Die Landschnecke Discus ruderatus im Postglazial Süddeutschlands. - Mitt. bayer. Staatssamml. Paläont. hist. Geol., 7: 135-154, 1 Abb.; München. 
EISSELE, K. \& SCHÄDEL, K. (1957): Neue Höhenschotterfunde auf dem Galgenberg bei Tübingen. - Jh. Ver. vaterl. Naturkde., 112, 1: 177-187, 8 Abb., 1 Tab.; Stuttgart.

Evans, J. G. (1972): Land Snails in Archaeology. - 436 S., 145 Abb., 17 Tab.; London-New York (Seminar Press).

FrAAS, E. (1896): Pleistozäne Bildungen im schwäbischen Unterlande. - Z. dt. geol. Ges., 48: 696-702, 2 Abb.; Stuttgart.

FRECHEN, J. (1975): Tephrostratigraphische Abgrenzung des Würmlösses und der älteren Lösse im Quartärprofil der Tongrube Kärlich, Neuwieder Becken. - Decheniana, 127: 157-194, 12 Abb., 7 Tab.; Bonn.

GEYER, D. (1912): Die Molluskenfauna der diluvialen und postdiluvialen Kalktuffe des Diessener Tales, eine biologisch-geologische Studie. - Mitt. geol. Abt. kgl. württ. stat. Landesamt, 9: 1-55, 2 Taf.; Stuttgart.

- (1913): Beiträge zur Kenntnis des Quartärs in Schwaben. - Jh. Ver. vaterl. Naturkde. Württ., 69: 277-302, 1 Abb.; Stuttgart.

- (1914): Über diluviale Schotter Schwabens und ihre Molluskenreste. - Jber. Mitt. oberrh. geol. Ver., N. F., 4: 120-138, 2 Taf.; Stuttgart.

- (1915): Die Mollusken der schwäbischen Kalktuffe. Jh. Ver. vaterl. Naturkde. Württ., 71: 55-114; Stuttgart.

- (1920): Die Mollusken des Cannstatter Sauerwasserkalks. - Jber. Mitt. oberrh. geol. Ver., N. F., 9: 61-66; Stuttgart.

- (1923): Die Quartärmollusken und die Klimafrage. Paläontol. Z., 5: 72-94; Berlin.

- (1927): Unsere Land- und Süßwasser-Mollusken. 224 S., 33 Taf.; Stuttgart (K. G. Lutz).

Held, A. (1913): Höhenschotter und Hochterrasse bei Rottenburg a. N. - Diss. Univ. Tübingen: 54 S.; Tübingen.

Heller, W. (1961): Die Talgeschichte des Neckars und seiner Zubringer zwischen Mittelstadt und Köngen. Diss. Univ. Tübingen; 175 S., 44 Abb.; Tübingen.

JAECKEL, S. G. A. (1962): Ergänzungen und Berichtigungen zum rezenten und quartären Vorkommen der mitteleuropäischen Mollusken. - In: P. BROHMER, P. EHRMANN \& G. Ulmer: Die Tierwelt Mitteleuropas 2,1 (Ergänzung): 25-294, 9 Taf.; Leipzig (Quelle \& Meyer).

Kranz, W., Berckhemer, F. \& WÄGele, H. (1930): Die "Seelberg"-Ablagerungen in Cannstatt, ihre geologischen Verhältnisse, Fossilfunde und Naturdenkmale. Veröff. Staatl. St. Natursch. württ. Landesamt Denkmalpfl., 7: 95-126, 5 Abb.; Stuttgart (Beiheft Jh. Ver. vaterl. Naturkde. Württ., 86).
LÖHR, H. \& BRUNNACKER, K. (1974): Metternicher und Eltviller Tuffhorizont im würmglazialen Löß am Mittelund Niederrhein. - Notizbl. hess. L.-Amt Bodenforsch., 102: 168-190, 2 Abb., 1 Tab.; Wiesbaden.

LOŽEK, V. (1964): Quartärmollusken der Tschechoslowakei. - Rozpravy Úst. úst. geol., 31: 373 S., 91 Abb., 32 Taf.; Prag.

- (1969): Über die malakozoologische Charakteristik der pleistozänen Warmzeiten mit besonderer Berücksichtigung des letzten Interglazials. - Ber. dt. Ges. geol. Wiss., A, Geol. Paläont., 14: 439-469, 10 Abb.; Berlin.

- (1976): Klimaabhängige Zyklen der Sedimentation und Bodenbildung während des Quartärs im Lichte malakozoologischer Untersuchungen. - Rozpravy Ceskosl. Akad. Ved, Rada Matem. Prírod. Ved, 86: 1-97, 7 Abb., 9 Tab., 10 Taf.; Prag.

MADER, M. (1978): Die Flußgeschichte des Neckars und das Wandern des Albtraufs. - Veröffentl. Naturschutz Landespflege Baden-Württemberg, 47/48: 433-507; Karlsruhe.

MAECK, H. S. (1963): Die Entstehungsgeschichte der interglazialen Kalktuffe des Dießener Tales bei Horb/Neckar. - Diss. Univ. Tübingen: 63 S., 15 Abb., 2 Tab., 1 Beil.; Sittard.

Mägdefrau, K. \& MaecK, H. S. (1965): Die fossile Pflanzen- und Tierwelt des interglazialen Kalktuffs von Dießen bei Horb/Neckar. - Fundber. aus Schwaben, N. F., 17: 237-249, 2 Abb., 2 Tab., 1 Taf.; Stuttgart.

MÜHLFELD, R. (1953): Der Bau der Rottenburger Flexur und die Neckarablagerungen bei Rottenburg. - Diss. Univ. Tübingen: 64 S., 6 Phot., 15 Abb.; Tübingen.

MÚNZING, K. (1966): Quartäre Molluskenfaunen aus BadenWürttemberg. - Jh. geol. Landesamt Baden-Württemberg, 8: 47-62, 8 Tab.; Freiburg i. Br.

- (1968): Molluskenfaunen aus altpleistozänen Neckarablagerungen. - Jh. geol. Landesamt Baden-Württemberg, 10: 105-119; 3 Tab.; Freiburg i. Br.

- (1971): Pleistozäne Molluskenfaunen aus dem Heilbronner Raum. - Jh. geol. Landesamt Baden-Württemberg, 13: 151-163, 1 Abb., 3 Tab.; Freiburg i. Br.

- (1973): Beiträge zur quartären Molluskenfauna BadenWürttembergs. - Jh. geol. Landesamt Baden-Württemberg, 15: 161-185, 5 Abb., 7 Tab.; Freiburg i. Br.

RÄHLE, W. (1983): Die Mollusken der Grabung Helga-Abri bei Schelklingen mit einer Anmerkung zum Fund einiger mesolithischer Schmuckschnecken. - Archäol. Korrespondenzbl., 13: 29-36, 3 Abb., 1 Tab.; Mainz.

ReIFF, W. (1965): Das Alter der Sauerwasserkalke von Stuttgart - Münster - Bad Cannstatt - Untertürkheim. Jber. Mitt. oberrh. geol. Ver., N. F., 47: 111-134, 3 Abb.; Stuttgart. 
REIFF, W. (1973): Bericht über die von der DEUQUATagung 1972 durchgeführte Exkursion zu den Travertinen von Stuttgart. - Eiszeitalter u. Gegenwart., 23/24: 410-412, 1 Tab.; Öhringen/Württ.

REMY, H. (1968): Zur Stratigraphie und Klimaentwicklung des jüngeren Pleistozäns in Mittel- und Westeuropa unter besonderer Berücksichtigung des Lösses. - Decheniana, 121: 121-145, 4 Abb., 5 Tab.; Bonn.

Schmid, A. (1924): Vom Alter der Neckartalrandstufen in Schwaben. - Jber. Mitt. oberrh. geol. Ver., N. F. 13: 119-124; Stuttgart.

- (1932): Erl. geol. Spezialkt. Württ. Bl. Rottenburg Bietenhausen (Nr. 108). - 49 S., 2 Taf.; Stuttgart (Kohlhammer).
Semmel, A. (1968): Studien über den Verlauf jungpleistozäner Formung in Hessen. - Frankf. geogr. H., 45: 133 S., 35 Abb.; Frankfurt a. M.

- (1969): Bemerkungen zur Wurmlößgliederung im Rhein-Maingebiet. - Notizbl. hess. L.-Amt Bodenforsch., 97: 395-399, $1 \mathrm{Abb}$.; Wiesbaden.

StEusLoff, U. (1953): Wanderungen und Wandlungen der Süßwasser-Mollusken Mitteleuropas während des Pleistozäns. - Arch. Hydrobiol., 48: 210-236, 4 Abb., 1 Tab.; Stuttgart.

Manuskript eingegangen am 29.11. 1985 
Proben-Nr.:

2

3

4

5

6

7

8

9

10

11

1. Schattenliebende Arten (Bewohner von Wäldern, Gebüschen etc.)

\begin{tabular}{|c|c|c|c|c|c|c|c|c|c|c|c|c|}
\hline$!$ & Aegopinella spec. & - & 1 & - & - & - & 1 & 2 & 1 & 3 & - & - \\
\hline * & Arianta arbustorum & 1 & 1 & 2 & 2 & 2 & 1 & 1 & 1 & 1 & 1 & 1 \\
\hline (!) & Bradybaena fruticum & 1 & - & 5 & 2 & 10 & 3 & 3 & 10 & 6 & 1 & - \\
\hline$!$ & Cepaea spec. & 1 & - & 1 & - & 2 & 2 & 2 & 2 & 1 & 1 & - \\
\hline * & Clausilia dubia & 2 & 1 & 1 & - & 1 & 1 & 3 & 4 & 4 & - & - \\
\hline$!$ & Cocblodina spec. & - & 1 & - & - & 4 & 2 & - & - & - & - & - \\
\hline (!) & Discus rotundatus & - & - & - & - & - & - & - & - & - & 2 & - \\
\hline (!) & Discus ruderatus & 4 & 2 & 4 & 1 & 6 & 8 & 9 & 6 & 9 & - & - \\
\hline$!$ & Ena montana & 1 & 1 & 2 & - & 3 & 1 & 2 & 2 & 4 & - & - \\
\hline * & Eucobresia diaphana & - & - & - & - & - & - & - & - & - & 1 & - \\
\hline$!$ & Helicodonta obvoluta & - & - & - & - & - & - & 1 & 1 & 1 & - & - \\
\hline$!$ & Macrogastra plicatula & 1 & - & - & - & 1 & - & 1 & - & 1 & 1 & - \\
\hline$!$ & Orcula doliolum & - & - & - & - & - & - & - & - & - & 1 & - \\
\hline * & Trichia villosa & 2 & - & 1 & - & 1 & - & 1 & 4 & 5 & 1 & - \\
\hline (!) & Vertigo pusilla & - & - & - & 2 & 1 & - & 1 & 1 & - & - & - \\
\hline * & Vitrea crystallina & 8 & 6 & 11 & - & 10 & 9 & 3 & 8 & 14 & 2 & - \\
\hline$!$ & Vitrea subrimata & - & 1 & - & - & - & - & - & 1 & - & - & - \\
\hline & & $\begin{array}{c}21 \\
(5,4 \%)\end{array}$ & $\begin{array}{c}14 \\
(4,6 \%)\end{array}$ & $\begin{array}{c}27 \\
(4,2 \%)\end{array}$ & $\begin{array}{c}7 \\
(2,1 \%)\end{array}$ & $\begin{array}{c}41 \\
(2,6 \%)\end{array}$ & $\begin{array}{c}28 \\
(5,7 \%)\end{array}$ & $\begin{array}{c}29 \\
(5,5 \%)\end{array}$ & $\begin{array}{c}41 \\
(7,0 \%)\end{array}$ & $\begin{array}{c}49 \\
(7,9 \%)\end{array}$ & $\begin{array}{c}11 \\
(16,2 \%)\end{array}$ & $\begin{array}{c}1 \\
(0,2 \%)\end{array}$ \\
\hline
\end{tabular}




\section{Proben-Nr.:}

1

2

3

4

2. Ubiquisten (Bewohner von Wäldern, Gebüsch und offenem Gelände)

\begin{tabular}{|c|c|c|c|c|c|c|c|c|c|c|c|c|}
\hline * & Abida secale & - & - & - & - & 1 & - & - & - & 1 & - & - \\
\hline$!$ & Carychium tridentatum & 6 & 7 & 21 & 2 & 17 & 35 & 8 & 14 & 7 & 5 & - \\
\hline * & Clausilia parvula & 4 & 1 & 5 & - & 8 & 3 & 5 & 5 & 4 & 1 & 3 \\
\hline * & Cocblicopa cf. lubrica & 9 & 7 & 19 & 3 & 19 & 11 & 9 & 19 & 27 & 2 & 1 \\
\hline (!) & Cocblicopa lubricella & - & - & 1 & - & - & - & - & - & 2 & - & - \\
\hline * & Euconulus fulvus & 3 & - & 1 & - & 3 & 1 & 2 & 1 & 1 & - & - \\
\hline (!) & Euomphalia strigella & - & 1 & - & - & 1 & - & - & - & - & - & - \\
\hline * & Limacidae/Agriolimacidae & 2 & - & - & - & 2 & 2 & 1 & 1 & 1 & - & 20 \\
\hline * & Nesovitrea hammonis & 4 & 11 & 30 & 4 & 32 & 14 & 16 & 29 & 34 & 6 & - \\
\hline * & Punctum pygmaeum & 1 & 6 & 5 & 3 & 13 & 7 & 5 & 8 & 1 & - & - \\
\hline * & Succinea oblonga & - & 1 & 1 & - & 14 & - & 2 & 5 & 7 & - & 220 \\
\hline * & Trichia bispida & 7 & 6 & 27 & 3 & 35 & 15 & 19 & 25 & 25 & 1 & 23 \\
\hline * & Vertigo substriata & - & - & 1 & - & - & 1 & - & - & - & - & - \\
\hline$!$ & Vitrea contracta & - & - & - & - & 1 & - & - & - & - & - & - \\
\hline & & 36 & 40 & 111 & 15 & 146 & 89 & 67 & 107 & 110 & 15 & 267 \\
\hline & & $(9,2 \%)$ & $(13,2 \%)$ & $(17,1 \%)$ & $(4,6 \%)$ & $(9,1 \%)$ & $18,0 \%)$ & $(12,7 \%)$ & $(18,3 \%)$ & $(17,8 \%)$ & $(22,0 \%)$ & $(62,1 \%)$ \\
\hline
\end{tabular}




\section{Proben-Nr.:}

12

3

45

6

7

8

$9 \quad 10$

11

3. Offenlandarten (Bewohner von Grasfluren und Felssteppen)

\begin{tabular}{|c|c|c|c|c|c|c|c|c|c|c|c|c|}
\hline+ & Columella columella & - & - & - & - & - & - & - & - & - & - & 28 \\
\hline * & Helicopsis striata & - & - & - & - & 3 & 1 & 2 & - & 1 & - & 5 \\
\hline+ & Pupilla loessica & - & - & - & - & - & - & - & - & - & - & 12 \\
\hline * & Pupilla muscorum & 3 & 2 & 5 & 1 & 4 & 18 & 35 & 1 & 9 & - & 107 \\
\hline * & Vallonia costata & 55 & 73 & 202 & 30 & 322 & 166 & 190 & 193 & 228 & 9 & 10 \\
\hline * & Vallonia pulchella & 16 & 19 & 70 & 3 & 77 & 42 & 36 & 51 & 66 & 22 & - \\
\hline (!) & Vertigo pygmaea & - & 3 & 4 & - & 5 & 3 & 1 & 1 & - & - & - \\
\hline & & 74 & 97 & 281 & 34 & 411 & 230 & 264 & 246 & 304 & 31 & 162 \\
\hline & & $(19,0 \%)$ & $(31,9 \%)$ & $(43,3 \%)$ & $(10,3 \%)$ & $(25,7 \%)$ & $(46,6 \%)$ & $(50,1 \%)$ & $(42,1 \%)$ & $(49,1 \%)$ & $(45,6 \%)$ & $(37,7 \%)$ \\
\hline
\end{tabular}

\section{Zeichenerklärung}

$+\quad$ Leitarten für Kaltzeiten (Glaziale)

* klimatisch indifferente Arten

(!) vorwiegend warmzeitliche Arten (auch in milderen Abschnitten der Kaltzeiten)

! warmzeitliche Arten

!! Leitarten für Warmzeiten (Interglaziale) 
4. Wasser- und Sumpfmollusken

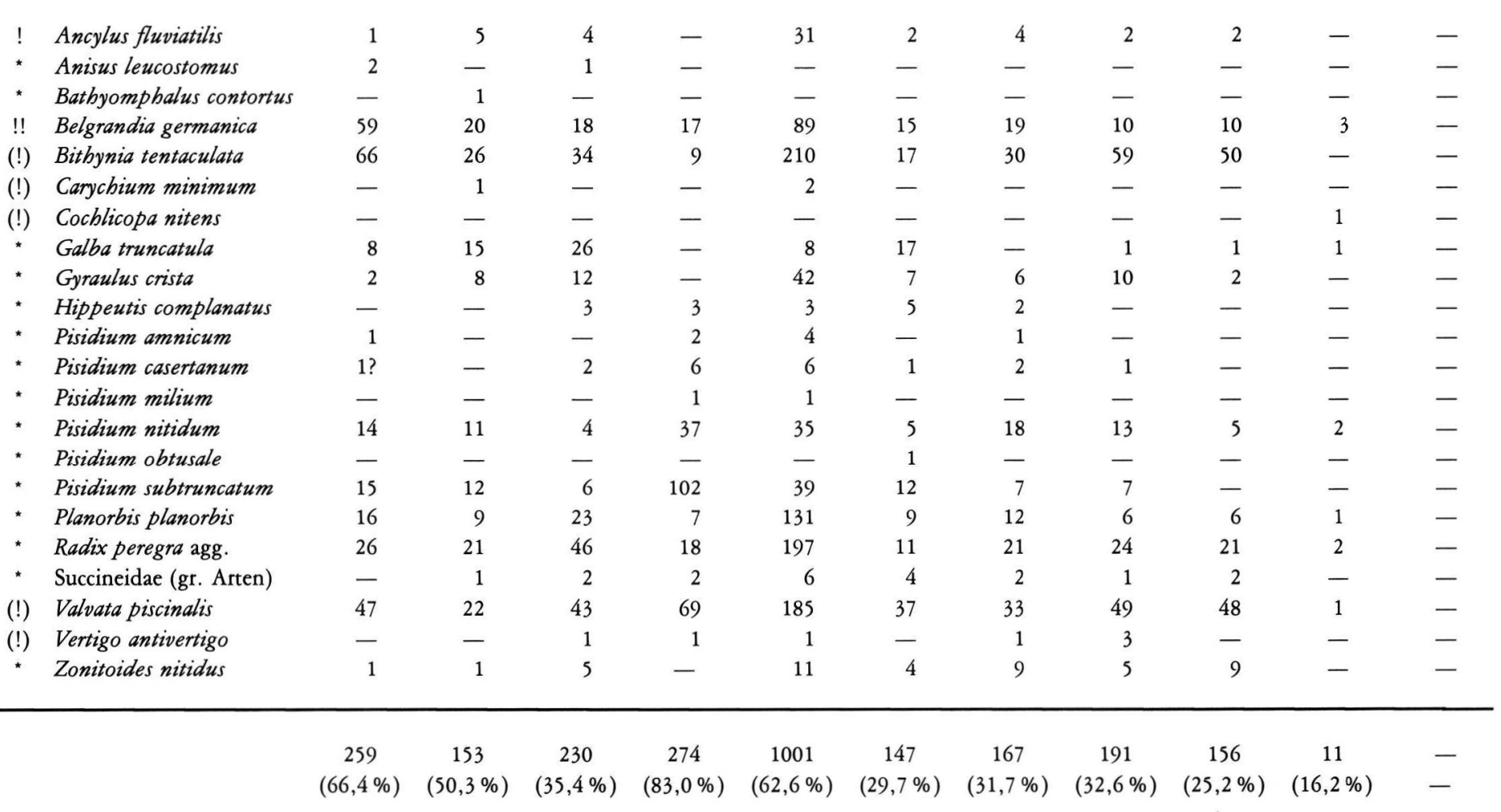

$\begin{array}{lrrrrrrrrrrr}\text { Individuenzahl }(=100 \%): & 390 & 304 & 649 & 330 & 1599 & 494 & 527 & 585 & 619 & 68 & 430 \\ \text { Artenzahl: } & 34 & 34 & 38 & 25 & 46 & 38 & 41 & 39 & 37 & 23 & 11\end{array}$


Tab. 2: Die Mollusken der Straßenbau-Aufschlüsse am Südrand von Heilbronn-Böckingen. Numerierung und Entnahme der Proben entsprechend Abb. 5.

Zeichenerklärung in Tabelle 1

\begin{tabular}{|c|c|c|c|c|c|c|c|c|c|c|c|c|}
\hline Proben-Nr.: & $1 / 2$ & $3 / 4$ & $5 / 6$ & 7 & 8 & 9 & 10 & 11 & 12 & 13 & 14 & 15 \\
\hline
\end{tabular}

1. Schattenliebende Arten (Bewohner von Wäldern, Gebüschen etc.)

\begin{tabular}{|c|c|c|c|c|c|c|c|c|c|c|c|c|c|}
\hline$!$ & Aegopinella spec. & - & 3 & 2 & 6 & 2 & - & - & - & - & - & - & - \\
\hline * & Arianta arbustorum & 2 & 1 & 2 & 1 & 1 & - & 1 & 1 & - & 1 & 1 & - \\
\hline$(!)$ & Bradybaena fruticum & 2 & 4 & 5 & 3 & 3 & - & - & - & - & - & - & - \\
\hline$(!)$ & Clausilia bidentata & - & - & - & - & 1 & - & - & - & - & - & - & - \\
\hline * & Clausilia pumila & 4 & 4 & 7 & 3 & 4 & - & 3 & 2 & - & - & - & - \\
\hline (!) & Discus rotundatus & - & 1 & - & - & - & - & - & - & - & - & - & - \\
\hline * & Eucobresia diaphana & - & - & 1 & - & - & - & - & - & - & - & - & - \\
\hline$!$ & Macrogastra plicatula & $1 ?$ & - & 1 & - & - & - & - & - & - & - & - & - \\
\hline$! !$ & Ruthenica filograna & - & 1 & 1 & 1 & 1 & - & 2 & - & - & - & - & - \\
\hline$(!)$ & Vertigo pusilla & - & - & - & - & 1 & - & - & - & - & - & - & - \\
\hline * & Vitrea crystallina & - & - & 9 & 2 & 1 & - & - & - & - & - & - & - \\
\hline
\end{tabular}

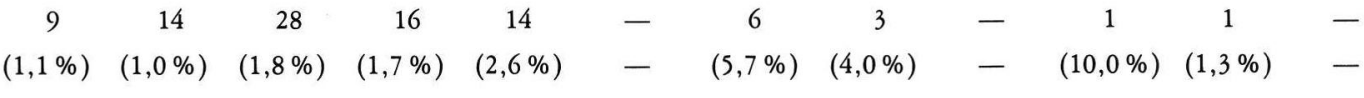


Fortsetzung Tabelle 2

\begin{tabular}{|c|c|c|c|c|c|c|c|c|c|c|c|c|}
\hline Proben-Nr.: & $1 / 2$ & $3 / 4$ & $5 / 6$ & 7 & 8 & 9 & 10 & 11 & 12 & 13 & 14 & 15 \\
\hline \multicolumn{13}{|c|}{ 2. Ubiquisten (Bewohner von Wäldern, Gebüsch und offenem Gelände) } \\
\hline * Abida secale & - & - & - & 1 & - & - & - & - & - & - & - & - \\
\hline ! Carychium tridentatum & - & - & 1 & - & - & - & - & - & - & - & - & - \\
\hline * Clausilia parvula & 1 & 1 & - & - & 2 & - & - & - & - & - & - & - \\
\hline * Cocblicopa cf. lubrica & - & 1 & 5 & 7 & 2 & - & 1 & - & - & - & - & - \\
\hline * Limacidae/Agriolimacidae & 7 & 24 & 42 & 34 & 25 & - & 3 & 4 & 6 & 5 & 5 & 11 \\
\hline * Nesovitrea hammonis & - & - & 1 & - & - & - & - & - & - & - & - & - \\
\hline * Punctum pygmaeum & - & 7 & 17 & 2 & - & - & - & - & - & - & - & - \\
\hline * Succinea oblonga & 5 & 6 & 48 & 3 & 8 & - & 15 & 8 & 1 & 2 & 41 & 382 \\
\hline * Trichia bispida & 83 & 80 & 125 & 64 & 31 & - & 22 & 6 & 1 & 1 & 4 & 26 \\
\hline \multirow[t]{4}{*}{ (!) Vertigo angustior } & - & - & 1 & - & 1 & - & - & - & - & - & - & - \\
\hline & 96 & 119 & 240 & 111 & 69 & - & 41 & 18 & 8 & 8 & 50 & 419 \\
\hline & $(12,1 \%)$ & $(8,6 \%)$ & $(15,7 \%)$ & $(11,9 \%)$ & $(12,7 \%)$ & - & $(38,7 \%)$ & $(24,0 \%)$ & $(88,9 \%)$ & $(80,0 \%)$ & $(64,9 \%)$ & $62,7 \%)$ \\
\hline & & & & & & & & & & \multicolumn{3}{|c|}{ Fortsetzung siehe nächste Seite } \\
\hline
\end{tabular}


Fortsetzung Tabelle 2

\begin{tabular}{lllllllllllll}
\hline Proben-Nr.: & $1 / 2$ & $3 / 4$ & $5 / 6$ & 7 & 8 & 9 & 10 & 11 & 12 & 13 & 14 & 15 \\
\hline
\end{tabular}

3. Offenlandarten (Bewohner von Grasfluren und Felssteppen)

\begin{tabular}{|c|c|c|c|c|c|c|c|c|c|c|c|c|c|}
\hline * & Chondrula tridens & 5 & 4 & 13 & 11 & 11 & - & 11 & 22 & 1 & - & 1 & 1 \\
\hline+ & Columella columella & - & - & - & - & - & - & - & - & - & - & 1 & 6 \\
\hline * & Granaria frumentum & - & - & 1 & - & - & - & 4 & 2 & - & - & - & - \\
\hline * & Helicopsis striata & - & - & - & - & - & - & - & 2 & - & - & - & 1 \\
\hline * & Pupilla muscorum & 30 & 25 & 46 & 13 & 12 & - & 5 & 3 & - & - & 23 & 236 \\
\hline (!) & Truncatellina cylindrica & - & 1 & 2 & 4 & 1 & - & 1 & - & - & - & - & - \\
\hline * & Vallonia costata & 152 & 592 & 621 & 407 & 255 & - & 24 & 22 & - & 1 & 1 & 4 \\
\hline * & Vallonia pulchella & 303 & 561 & 510 & 315 & 145 & - & 11 & 2 & - & - & - & 1 \\
\hline (!) & Vertigo pygmaea & 1 & 27 & 49 & 50 & 25 & - & 2 & 1 & - & - & - & - \\
\hline
\end{tabular}

\begin{tabular}{|c|c|c|c|c|c|c|c|c|c|c|c|}
\hline 491 & 1210 & 1242 & 800 & 449 & - & 58 & 54 & 1 & 1 & 26 & 249 \\
\hline $61,8 \%)$ & $(87,5 \%)$ & $(80,9 \%)$ & $(85,9 \%)$ & $(82,7 \%)$ & - & $(54,7 \%)$ & $(72,0 \%)$ & $(11,1 \%)$ & $(10,0 \%)$ & $(33,8 \%)$ & $(37,3 \%)$ \\
\hline
\end{tabular}




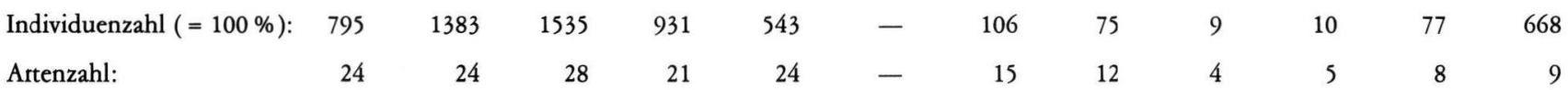


